\title{
La construction d'une communauté de sécurité en Europe : le cas des pays nordiques
}

Cyril Coulet, Nicolas Wuest-Famose et Louis Clerc

\section{(2) OpenEdition \\ 1 Journals}

\section{Édition électronique}

URL : http://journals.openedition.org/conflits/963

DOI : $10.4000 /$ conflits.963

ISSN : $1777-5345$

Éditeur :

CCLS - Centre d'études sur les conflits lilberté et sécurité, L'Harmattan

\section{Édition imprimée}

Date de publication : 1 septembre 2003

Pagination : 97-126

ISBN : 2-7475-5377-9

ISSN : 1157-996X

\section{Référence électronique}

Cyril Coulet, Nicolas Wuest-Famose et Louis Clerc, «La construction d'une communauté de sécurité en Europe : le cas des pays nordiques », Cultures \& Conflits [En ligne], 51 | automne 2003, mis en ligne le 02 février 2004, consulté le 30 mars 2021. URL : http://journals.openedition.org/conflits/963 ; DOI https://doi.org/10.4000/conflits.963

Ce document a été généré automatiquement le 30 mars 2021.

Creative Commons License 


\title{
La construction d'une communauté de sécurité en Europe : le cas des pays nordiques
}

\author{
Cyril Coulet, Nicolas Wuest-Famose et Louis Clerc
}

1 Dans les pays européens, la sécurité intérieure est du ressort de différents types de police $^{1}$. C'est un legs de l'histoire et des traditions de chacun des Etats mais ce legs aura incontestablement des répercussions lorsqu'il s'agira de traiter, plus avant, de la sécurité à l'échelle de l'Europe.

2 Au plan militaire, l'Europe se dote progressivement de capacités qui devraient répondre, à terme, à l'ensemble des missions dites de Petersberg à savoir : les missions d'action humanitaire et d'évacuation, les missions de maintien de la paix et les missions de force de combat pour la gestion des crises sans oublier depuis peu, la lutte contre le terrorisme. Pour ce qui relève des activités de police en projection extérieure, l'idée qui consisterait à rétablir l'ordre s'il est troublé et à exercer une surveillance des différentes zones abritant des trafics illicites est très largement répandue. Et au-delà d'un tel «domaine d'activité ", se situant à l'interface du deuxième et du troisième pilier de l'Union européenne, la consolidation de la paix après son rétablissement et la (re)construction des Etats de droit, revêtent une acuité nouvelle.

3 Le rétablissement de la police judiciaire, de la justice civile et de la justice pénale, ainsi que la formation des polices locales sont des missions incontournables. Si l'on souhaite que soient restaurés ou même instaurés - de manière durable - paix et Etat de droit, il faut que la confiance de la population soit au rendez-vous. Or, celle-ci dépend de l'efficacité et de l'éthique de ces institutions. Qui doit avoir la charge temporaire de se substituer à elles et de les former est une question importante. Les polices civiles à statut militaire sont, semble-t-il, mieux placées dans ce rôle. Certains pays comme l'Italie ou la France ont confié à leurs forces de police placées sous un statut militaire (respectivement les Carabiniers et les Gendarmes) des missions de maintien de l'ordre, tout en étant capables d'assurer des tâches de renseignement policier et de police judiciaire. 

de sécurité et de défense s'est principalement concentré sur son aspect militaire, à savoir la force de réaction rapide. A ce jour, en revanche, peu d'attention est consacrée à sa contrepartie dite civile : la gestion des crises non-militaires ou civiles et la force de police de réaction rapide qui lui est attachée.

Cet article expose la manière dont les pays nordiques (Danemark, Finlande, Norvège, Suède) envisagent ces problèmes. En effet: quels seront les types d'objectifs et d'actions que ces pays accepteront que leurs polices à statut civil remplissent, et quels seront ceux qu'ils refuseront de les voir remplir? Quels types d'unités sont-ils susceptibles de déployer dans des opérations extérieures? Comment intercaler des polices à statut civil dans un "environnement " plus militarisé, avec les problèmes de subordination, de commandement ou de cadre juridique que cela pourrait soulever?

6 Si la participation des pays nordiques en termes de moyens logistiques et humains est pour l'instant limitée, il n'en demeure pas moins qu'un débat public dans ces pays sur la légitimité de certaines interventions pourrait avoir des répercussions sur l'ensemble des pays membres de l'Union européenne. Il parait donc important d'étudier l'organisation, les motivations et les intérêts des pays nordiques dans les domaines intéressant les missions de Petersberg, à savoir des missions de police internationale, de consolidation de la paix et de rétablissement du droit.

7 Tableau de situation des quatre pays étudiés (au 1er juillet 2002)

8 DANEMARK FINLANDE NORVEGE SUEDE Capitale Copenhague Helsinki Oslo Stockholm Régime politique Monarchie constitutionnelle République Monarchie constitutionnelle Monarchie constitutionnelle Chef de l'Etat Margrethe II Tarja Halonen Harald V Carl XVI Gustav Chef

de gouvernement Anders Fogh Rasmussen Paavo Lipponen Kjell Magne Bondevik Goran Persson

Population 5352815517578345034408875053 PNB (euros) 136,2 milliards 118,3 milliards 124,1 milliards 197 milliards PNB/hab. 25500229002770022200 Neutralité Non Oui Non Oui ONU Oui Oui Oui Oui OTAN Oui Non Oui Non UE Oui Oui Non Oui UEO Oui Oui Membre associé Oui Police à statut civil Oui Oui Oui Oui Police à statut militaire Non Oui (le corps des gardes-frontières/ gardes-côtes) Non Non Effectifs de police en uniforme 100008000

+3 102(gf/gc) 1070015858 Ratio 1 policier/x habitants 1/535 1/466 1/421 $1 / 559$ Policiers en

opérations

extérieures 734668101 Participation à la capacité européenne

de police 12575

soit 70 policiers civils et 5 gardes frontières 80170 Effectifs des forces armées (appelés compris) 29212435003100041000 Service national obligatoire Oui oui Oui oui Ratio 1 soldat/x habitants 1/183 1/119 1/145 1/216 Soldats en

opérations

extérieures 1270101713601995

Le Danemark 
18 Structurellement, l'organisation de la sécurité intérieure danoise se caractérise par le rôle prépondérant du ministère de la Justice et par l'étroite imbrication qui existe entre l'appareil policier et l'appareil judiciaire. Aussi, l'unique force de police est-elle rattachée au ministère de la Justice.

19 Les dispositions statutaires fondamentales qui gouvernent le travail de la police se trouvent à l'article 108 de la loi sur l'administration de la Justice: les devoirs de la police $^{2}$ sont de maintenir la sécurité, la paix et l'ordre ; de s'assurer du bon respect des lois et règlements; de prendre les mesures nécessaires pour empêcher tout crime ; d'enquêter sur les crimes et de poursuivre les contrevenants; les autres devoirs peuvent être imposés à la police avec l'autorisation du ministre de la Justice.

20 La police, tant au Danemark qu'aux Féroé et au Groenland, n'est constituée que d'une seule entité nationale, directement employée par l'Etat.

21 Aux yeux des autorités danoises, il est important que les candidats qui souhaitent rejoindre les rangs de la police soient représentatifs de toute la diversité de la population danoise. Aussi, la police est-elle ouverte aux hommes comme aux femmes, ayant des origines sociales, culturelles et ethniques différentes ${ }^{3}$.

22 Les policiers danois portent traditionnellement une arme à feu dans l'exercice de leurs fonctions. Mais l'usage des armes à feu est strictement réglementé. Par ailleurs, la police danoise semble bien préparée à faire face à des manifestations violentes. En effet, celle-ci a dû gérer ces dernières années quelques manifestations violentes dans les rues de Copenhague (en particulier suite à des consultations populaires sur la participation du Danemark à la construction de l'Union européenne). Le détachement de police en charge de la lutte anti-émeute est exclusif à la police de Copenhague. L'efficacité de celui-ci s'explique non seulement par son équipement, mais aussi par l'utilisation impressionnante des chevaux.

23 La vigueur des manifestions anti-mondialisation ou des mouvements contestataires lors des sommets européens comme à Göteborg ou du G8 comme à Gênes ont décidé les autorités danoises à prendre la question de la sécurité à bras le corps compte tenu de la présidence de l'Union européenne par le Danemark durant la deuxième moitié de 2002.

24 En effet, pour toute la durée de la présidence danoise, la ministre de la Justice Lene Espersen a prévu d'utiliser près de 100 millions d'euros pour la mobilisation de la police " afin d'empêcher toute attaque terroriste ou des manifestations violentes "4.

Qu'en est-il des policiers lorsqu'ils sont intégrés à des missions internationales ? Selon les autorités policières danoises, il est assez délicat de réunir un nombre important de policiers prêts à se porter volontaires pour effectuer une mission en opération extérieure.

26 Les autorités souhaitent que les policiers qui partent en opération extérieure disposent déjà d'une solide expérience au Danemark et ne remplissent pas plus de trois fois une mission en opération extérieure. Aussi, ces policiers ont-il, pour beaucoup d'entre eux, plus de trente-cinq ans, sont mariés et ont des enfants et présentent des profils psychologiques certainement moins aventuriers ( less adventurous $»^{5}$ ) que les soldats.

27 Ce choix porté sur un tel profil de policiers explique peut-être la volonté de la police danoise de ne pas se voir imposer des missions autres que celles pour lesquelles elle a été envoyée en opération extérieure. Les Danois ne veulent pas déployer leurs policiers pour des missions non clairement définies et pour lesquelles subsistent des zones grises de responsabilités ou de compétences. Selon les policiers danois, il est indispensable 
qu'ils soient non-armés, qu'ils ne fassent pas de la gestion de foule et, surtout, qu'ils ne soient pas intégrés, d'une façon ou d'une autre, à un commandement militaire ${ }^{6}$. Les autorités de la police danoise sont parfaitement d'accord pour envoyer leurs hommes assurer des formations ou des missions de police civile, mais il est hors de question que la police soit confrontée à des «tâches plus difficiles » ${ }^{7}$. Au Kosovo, la police danoise est armée, mais le ministère de la Justice préfère de loin que cela ne se reproduise plus et que les soldats, avec leurs équipements, aient plutôt la charge de la gestion des foules ( « riot control » selon la terminologie danoise). Les autorités de police préfèrent que, petit à petit, les affaires de police soient transférées à la police civile, à mesure que le pays se pacifie; que cette police civile comprenne certes des éléments de police danoise, par exemple, mais qu'elle comprenne aussi et surtout des éléments de la gendarmerie qui, eux, sont équipés et entraînés pour faire face à des situations plus périlleuses ${ }^{8}$.

Les autorités de la police danoises ont, en outre, insisté sur deux caractères essentiels de leur police en opération extérieure : leur intégrité et leur rapide mobilisation (en moins de dix jours, un contingent de policiers danois peut être mobilisé).

Compte tenu d'effectifs militaires limités pour participer à des opérations extérieures, le Danemark préfère déployer ses soldats dans des missions en Europe (et donc plutôt dans la cadre de l'OTAN) pour délaisser dernièrement les opérations de l'ONU. L'Etatmajor danois ne tient plus vraiment à voir ses troupes participer à des missions d'observation ou d'intervention, mais préfère bien plutôt - compte tenu de son instrument militaire aujourd'hui performant comme la DIB - participer à des missions un peu plus « combatives "?.

Le changement réel d'orientation politique en matière de participation à des opérations militaires extérieures date de février 1998, au moment de la crise irakienne, lorsque le Danemark s'est prononcé en faveur d'une attaque contre l'Irak sans que le Conseil de sécurité de l'ONU ait donné son autorisation. Ce qui constituait une claire atteinte au droit international. Toujours est-il que rien de tout cela ne s'est produit : les opérations militaires ont eu lieu en décembre 1998 et le Danemark n'a pas été sollicité pour participer à cette opération, en dépit de son souhait d'y être associé. Dès 1997, l'Etat-major danois tire les leçons de sa participation à la mission IFOR ${ }^{10}$. Parmi les éléments retenus, les Danois pensent qu'un entraînement adéquat doit être imaginé pour faire face aux populations civiles ( handling of civilians ${ }^{11}$ ).

Le jeudi 17 juin 1999, le Parlement a donné son accord pour envoyer une contribution danoise à la KFOR. La force danoise consistait alors en un bataillon d'infanterie mécanisée, une structure de soutien logistique et du personnel pour le poste de commandement, soit un total approximatif de 850 hommes. Le bataillon est alors déployé sous le commandement de la brigade française, en charge du secteur géographique nord-ouest du Kosovo, proche de la ville de Kosovska Mitrovica.

La région de Mitrovica est l'une des plus instables du Kosovo et à de nombreuses occasions les soldats danois ont dû apporter leur soutien aux troupes françaises dans la ville même de Mitrovica afin de contrôler les foules. Les militaires français en opération à Mitrovica ont remarqué l'efficacité de l'armée danoise dans le maintien de l'ordre et la gestion des foules, en particulier par l'utilisation de ses unités de chiens. De leur côté, les forces armées danoises se sont félicitées de travailler de façon aussi proche avec les forces françaises ${ }^{12}$. 
- Une aide générale. Sont traitées sous cet aspect les questions relatives au transport des blessés, au transport d'organes vitaux, au matériel à apporter aux navires en détresse, à la recherche des personnes portées disparues, à la neutralisation de munitions, au déminage, au déneigement de la chaussée dans des situations difficiles, à la lutte contre l'incendie (si celui-ci est majeur) et à la régulation de la circulation sur la voie publique ;

39

- Une aide en cas de catastrophe. Les forces armées apportent leur soutien aux autorités civiles en cas de catastrophe majeure comme des accidents ferroviaires, des incendies de forêt, des pollutions d'hydrocarbure ;

40

- Une assistance spéciale à la police. Il s'agit d'un sujet où les textes en vigueur sont classés confidentiels. Concrètement, il s'agit de cas où le criminel est à ce point lourdement armé que les moyens dont la police dispose ne sont pas suffisants. Il s'agit aussi des attentats terroristes. Et enfin des « désordres intérieurs » devant lesquels les seules ressources de la police seraient insuffisantes pour restaurer l'ordre public. Mais cette formulation, il faut bien le reconnaître, demeure relativement floue.

41 L'intervention n'est possible qu'après autorisation du ministère de la Défense suite à l'avis conforme de l'Etat-major. La règle fondamentale en la matière stipule aux forces armées d'agir de la façon la plus discrète possible quand elles entrent en action. Dans la mesure du possible, les forces armées laissent à la police le soin de faire face aux 
manifestations violentes. De cette façon, les forces armées peuvent prendre à leur charge les fonctions que la police seule ne pourrait assurer dans une situation de crise violente comme, par exemple, la surveillance des bâtiments ou la sécurité des autorités. L'implication des forces armées dans une mission de rétablissement de l'ordre dans une situation insurrectionnelle n'est autorisée qu'en ultime recours. Les représentants de la police ont été très clairs sur ce point, estimant même impossible toute participation de l'armée à la sécurité intérieure. De leur côté, les autorités militaires danoises ont été un peu moins affirmatives ${ }^{14}$. Toutefois, dans la perspective du sommet européen de Copenhague de décembre 2002, il ne semble pas prévu que les forces armées prêtent leur concours aux forces de police en vue du maintien de l'ordre dans la capitale.

Ainsi, il apparaît que le maintien de l'ordre au Danemark est bien de la compétence de la police. Mais le recours aux forces armées est possible car il est juridiquement envisagé. Les militaires danois semblent assez réticents à l'idée de devoir agir et faire du maintien de l'ordre au Danemark. Ils assurent toutefois qu'ils le feront si l'ordre leur en est donné. La police de son côté semble totalement opposée à ce que l'armée puisse intervenir et la suppléer en cas de troubles graves.

Ce que la police est prête à faire à l'intérieur des frontières danoises, elle n'est pas prête à le faire en opération extérieure. Pour la police danoise, il est hors de question de faire du maintien de l'ordre hors des frontières danoises. Elle souhaite toutefois que dans les forces de police internationales chargées des missions dites de police civile sur des théâtres extérieurs, soient adjointes - en cas de nécessité - d'autres unités étrangères formées à la lutte anti-émeute telles que la gendarmerie nationale.

Avec une police danoise peu encline à vouloir faire du maintien de l'ordre (riot control) à l'extérieur, l'armée danoise s'y est préparée. Une formation des membres de la brigade internationale danoise est assurée. Ce savoir-faire, mis à l'épreuve avec succès à Mitrovica principalement, a fait la réputation des troupes danoises.

La Norvège

En l'absence de ministère de l'Intérieur, c'est le ministère de la Justice qui exerce son pouvoir de tutelle sur la police. Police civile, son activité est très strictement encadrée. Police démocratique, elle se veut aussi très proche des populations.

L'une des caractéristiques fondamentales de la police norvégienne est de n'être qu'une seule et unique force de police et d'être rattachée à un seul département ministériel.

D'une façon générale, les missions de la police norvégienne ne sont guère différentes de celles des autres pays européens, à savoir : assurer la protection des personnes et des biens, la tranquillité et l'ordre publics.

Et, lorsque les opérations de maintien de la paix ont commencé à changer de nature avec l'apparition de conflits internes plutôt qu'externes, la question de faire participer des officiers de police a immédiatement intéressé la Norvège.

La Norvège exprime ses réticences à participer à des opérations internationales de police civile sans mandat clair. Pour la Norvège, la priorité fondamentale dans une opération de police civile, c'est l'entraînement, la formation des polices locales. Les opérations que la Norvège privilégie sont des missions de formation, d'entraînement, d'assistance, de contrôle et de conseil auprès des autorités locales. Aussi, la Norvège ne voudrait s'engager dans une opération extérieure que lorsqu'il est apparent, dès le départ, que les autorités locales sont clairement identifiées et installées et que leur légitimité n'est pas mise en doute par les populations. 

comme risquée. La crise est plus complexe : la force de police internationale a vocation à agir prioritairement en substitution aux polices locales défaillantes par l'exécution de missions visant à la restauration de la sécurité publique (maintien de l'ordre, protection des biens et des personnes) et à la réactivation des organes judiciaires et pénitentiaires. Les autorités norvégiennes ont bien compris, au travers de l'exemple du Kosovo, que sa force de police n'était pas vraiment adaptée pour mettre en œuvre un « executive policing».

51 Les Norvégiens soutiendront prioritairement les opérations ONU, car elles sont à leurs yeux les plus légitimes, au détriment d'opérations OTAN ou Union européenne, organisations moins légitimes aux yeux de la population.

Pour les Norvégiens, il est tout à fait impensable que l'armée puisse être associée à la police dans ses missions de sécurité intérieure. Mais ce qui est vrai à l'intérieur des frontières de la Norvège, ne l'est pas forcément sur des théâtres d'opérations extérieures où l'armée sait remplir des missions de police. Il faut toutefois préciser qu'exceptionnellement et de façon très encadrée, l'armée peut être mise à la disposition de la police pour aider au maintien de la sécurité intérieure de la Norvège.

Les troupes norvégiennes sont envoyées sur des théâtres d'opération extérieure, soit de façon autonome, soit intégrées à la Brigade nordique. Après avoir, pendant de longues années, participé aux opérations d'interposition ou de maintien de la paix sous l'égide de l'ONU, la Norvège a dû faire face à de fortes demandes en troupes sur le théâtre européen. De fait, des troupes ont été retirées des contingents affectés aux opérations de l'ONU pour revenir à l'OTAN $^{15}$.

Les critiques de l'opinion publique ont cependant été vives, ne comprenant pas pourquoi les troupes norvégiennes étaient désengagées des opérations ONU au profit des opérations OTAN. Cette affectation différente des troupes norvégiennes n'a pas été sans créer des tensions entre le ministère des Affaires étrangères et le ministère de la Défense, le premier souhaitant envoyer des troupes à l'extérieur de façon disproportionnée par rapport aux moyens du pays, en particulier afin de ne pas entièrement découvrir l'ONU, compte tenu de l'accession de la Norvège, au 1er janvier 2001, à un siège de membre non-permanent au Conseil de sécurité à New York.

55 Pour le ministère de la Défense, il n'y a pas véritablement de motivation à participer à des opérations extérieures. De plus l'armée n'a aucune tradition historique dans ce domaine.

56 L'armée norvégienne est tout d'abord entraînée à des missions de type article $5 \mathrm{du}$ traité Atlantique. Puis, compte tenu de son activisme dans des opérations extérieures, les soldats norvégiens ont suivi un entraînement spécifique aux opérations de maintien de la paix. Aujourd'hui toutefois, avec l'extension des compétences sous commandement OTAN, l'armée norvégienne effectue des tâches pour lesquelles elle n'était pas préparée, à savoir le maintien de l'ordre. Sans aucune formation au départ, les militaires norvégiens ont tout d'abord appris "sur le tas", puis avec leurs expériences et quelques stages suivis à l'étranger, les Norvégiens ont constitué leurs propres méthodes d'apprentissage au maintien de l'ordre.

57 C'est ainsi que le bataillon de réaction immédiate reçoit une formation de base systématique au maintien de l'ordre. 

besoin des moyens logistiques des forces armées pour rechercher des criminels par exemple. Dans ce cas, les forces armées sont placées sous l'autorité et le contrôle de la police. Ces réquisitions sont aussi autorisées dans des situations d'urgence telles que des catastrophes naturelles, où là aussi, les moyens militaires peuvent être mis à la disposition du ministère de la Justice. Les gardes-côtes dépendent entièrement du ministère de la Défense (Marine royale). Et ceux-ci ont des tâches spécifiques en matière de police, en particulier depuis l'entrée en vigueur des Accords de Schengen, avec un contrôle strict de l'immigration.Une unité spécifique est chargée de la surveillance de la frontière avec la Russie : les gardes-frontières. Unité spécifique de l'armée de Terre, elle est en charge de la surveillance, du contrôle des entrées et de l'immigration sur le territoire norvégien. Elle n'a pas à proprement parler de pouvoirs de police. Aussi les gardes-frontières ne peuvent-ils procéder à des arrestations et ils sont obligés, pour cela, de remettre les délinquants à la police. La responsabilité des gardes-frontières comme du contrôle civil des personnes aux autres frontières de la Norvège est confiée au Commissaire aux Frontières. Celui-ci est traditionnellement issu de l'armée norvégienne, recruté au grade de colonel, il est placé sous la tutelle du ministère de la Justice. De plus, en vertu du code des douanes de 1966 (\$20), les forces armées sont obligées de venir en aide aux services douaniers lorsque ceux-ci le demandent et de les protéger dans l'exercice de leur fonction ${ }^{17}$.

62 La sécurité des installations pétrolières et gazières offshore, nombreuses pour la Norvège, est beaucoup plus simple : elle est assurée par le ministère de la Défense suite à un accord avec la ministère de la Justice. En fait, cela est dû à ses compétences antiterroristes. Cette compétence est technique et induit un contrôle strict de son action par la police. Les troupes d'élite formées à la lutte anti-terroriste dépendent elles aussi 
des forces armées, dites «forces spéciales ", elles comptent deux cents membres. Elles sont placées sous l'autorité et le contrôle du ministère de la Justice en cas d'intervention.

Plus anecdotique peut-être, mais de façon traditionnelle, la sécurité de la famille royale est partiellement assurée par l'armée. Le roi de Norvège dispose d'une garde d'apparat qui dispose toutefois des moyens techniques nécessaires pour assurer non seulement la sécurité du palais royal, mais aussi celle de la famille royale lors d'événements à caractère national.

Suite aux attentats du 11 septembre 2001, le discours officiel sur la répartition des compétences entre l'armée et la police sur le territoire norvégien semble changer. C'est tout d'abord le Premier ministre Kjell Magne Bondevik, qui le 8 novembre 2001 devant le Storting annonce : «dans la lutte contre le terrorisme (...) une coopération très étroite doit être établie entre la société civile et les forces de défense ». Puis, plus clairement encore, dans un discours tenu le 22 mars 2002 par la ministre de la Défense Kristin Krohn Devold, celle-ci annonce : " il y a très peu de raisons pour que la Norvège soit une cible du terrorisme international (...). C'est le ministère de la Justice qui a la responsabilité générale d'assurer la sécurité des Norvégiens en temps de paix. Cela signifie que le ministère de la Défense et nos forces armées coordonnent leur travail avec le ministère de la Justice d'une façon un peu plus active. Au début du mois d'avril, je soumettrai au Parlement une proposition relative à la politique de défense norvégienne. Elle apportera des changements nécessaires à la structure de notre défense et aux budgets qui lui sont liés (...). La proposition formule comment les forces armées doivent s'adapter aux nouveaux défis posés à notre politique de sécurité. Les forces armées ont besoin de devenir plus flexibles pour faire face à la menace terroriste ».

Le poids de l'opinion publique en Norvège, à l'instar des autres pays scandinaves, est extrêmement important. Or l'opinion publique semble plutôt réticente à l'idée de voir la Norvège s'investir sur la scène internationale ${ }^{18}$. Traditionnellement neutraliste, $a$ contrario des élites politiques et administratives, l'opinion publique soutient les opérations non-militaires de type humanitaire, placées sous l'autorité de l'ONU. Et si opérations de police il doit y avoir, celles-ci doivent être limitées soit à un entraînement ou à une formation des polices locales, soit à des opérations de police dites de proximité. Il n'en va pas de même pour des opérations menées par l'OTAN ou l'Union européenne et durant lesquelles les armes pourraient parler.

La Suède

66 La constitution de la force civile de l'Union européenne se heurte à des problèmes opérationnels en raison de la pluralité des approches dans la définition du statut et de l'emploi de ces forces. L'adoption de la proposition finno-suédoise de constitution d'une force européenne civile projetable lors du Conseil européen d'Helsinki en décembre 1999 a mis en lumière la difficile conciliation de modèles institutionnels de forces de sécurité en Europe. Il semble dès lors pertinent de s'interroger sur l'existence d'une approche particulière de la Suède sur les opérations extérieures de police. En effet, la création de forces de sécurité (forces de police et forces armées en l'occurrence) répond autant à des objectifs fonctionnels qu'à une logique institutionnelle de promotion d'un modèle de communauté de sécurité. La notion de communauté de sécurité dans ce texte se conforme à la définition opérée par Deutsch à savoir: "un groupe de personnes qui sont devenues intégrées [c'est-à-dire] ayant réalisé à l'intérieur d'un territoire un 'sens de la communauté' ainsi que des institutions et des pratiques suffisamment solides et répandues pour assurer sur le temps long, des probabilités raisonnables 
de 'changement pacifique' à l'intérieur de la population $»^{19}$. Autrement dit le modèle institutionnel dans lequel s'inscrivent les forces de sécurité ne répond pas seulement à un besoin fonctionnel mais il procède également du mode de constitution d'une société en communauté de sécurité. Le corollaire de cette assertion est que le modèle institutionnel varie selon des caractéristiques nationales dans la mesure où chaque communauté de sécurité définit ses modes de résolution du conflit dans des termes différents. Le modèle institutionnel imprime en retour sa marque sur la perception des opérations extérieures. Il va de soi que le modèle institutionnel concerne l'organisation de ces forces comme en témoigne l'abolition inédite du grade de sous-officier dans l'armée suédoise à partir de 1968. Il englobe également la répartition des compétences entre les forces de police et les forces armées ainsi que leurs doctrines d'emploi.

67 La nécessité de garantir l'acceptation de la forme institutionnelle des forces de sécurité a été particulièrement vive en Suède et la synthèse n'a été opérée qu'en 1940, à l'occasion de la mobilisation totale de la société suédoise pour sa défense organisée sous l'étendard de ce qu'il est convenu d'appeler «la neutralité suédoise ». La pierre angulaire de la définition et la répartition des compétences entre les forces de sécurité suédoises réside toutefois dans la loi relative à la création de la police nationale en 1933. Jusqu'à l'adoption de cette loi, la répartition des compétences était relativement simple puisque les forces de police, organisées à l'échelle des communes, assuraient toutes les missions de maintien de l'ordre sauf en cas de menaces d'émeutes auquel cas les autorités administratives régionales pouvaient faire appel aux forces armées. Toutefois ce modèle institutionnel a été fortement ébranlé consécutivement aux événements survenus à Ådalen en mai 1931. Une manifestation de grévistes désarmés fut arrêtée par l'armée qui ouvrit le feu et fit cinq morts. Le retentissement politique de cette affaire, dans un climat de fortes tensions sociales, fut tellement important qu'une commission d'enquête parlementaire fut créée. Cette dernière préconisa la constitution d'une force de police nationale pour suppléer aux forces armées en cas de défaillance des forces de police locales en mettant l'accent sur le fait que : «cela démontre avec force ce qui était déjà apparu, c'est-à-dire à quel point l'armée est un moyen inadapté pour maintenir l'ordre dans des situations où il n'est pas question de rencontrer une résistance ouvertement armée $»^{20}$.

L'un des éléments les plus surprenants du débat parlementaire fut l'opposition du parti social-démocrate à l'adoption de la loi portant création de la police nationale ${ }^{21}$. Cette opposition paraît d'autant plus surprenante que le parti social-démocrate détenait le monopole de la représentation des ouvriers qui avaient été les victimes de ces événements. Elle peut se comprendre au regard de l'attrait intellectuel qu'avait exercé sur les dirigeants sociaux-démocrates l'ouvrage de Jaurès intitulé : L'Armée nouvelle. Ainsi, Hjalmar Branting, dirigeant du parti social-démocrate, assura la traduction de cet ouvrage en suédois, publié en 1916 sous le titre: Les travailleurs, la patrie et l'armée(Arbetarna, fosterlandet och armén). Les forces armées paraissaient ainsi être l'instrument de la cohésion nationale en permettant l'intégration des différents groupes sociaux. De même, l'intégration des ouvriers devait tendre à contenir le "militarisme belliqueux" des forces armées. La troupe semblait dès lors moins dangereuse qu'une force de police nationale de 200 hommes dont le recrutement aurait pu exclure les ouvriers. La présence continue des sociaux-démocrates à la tête de la Suède de 1932 à 1976, à l'exception de quelques mois en 1936, a cependant éloigné le risque d'une police nationale transformée en milice aux mains du patronat. Dès lors, la stricte répartition des compétences entre les forces de police et les forces armées s'est 
perpétuée même s'il n'existe plus de police nationale spécialisée dans le maintien de l'ordre en Suède.

69 La prédominance des sociaux-démocrates a également contribué à reconstruire une communauté nationale, autour du concept de foyer du peuple (folkhem), que les controverses autour des questions de défense et les troubles sociaux avaient profondément désunie. En effet, le concept de folkhem a permis de supplanter le concept de nation qui, instrumentalisé par les conservateurs, avait conduit à la construction de la figure de l'ouvrier comme ennemi intérieur. La question de la construction de la communauté de sécurité nationale a donc dominé l'agenda politique suédois jusqu'à la seconde guerre mondiale parce qu'elle était le corollaire nécessaire de l'existence d'une communauté politique suédoise. Par ailleurs, les accords de Saltsjöbaden conclus entre le patronat et le syndicat LO en 1938 ont permis l'institution d'un dialogue social. Ainsi, la Suède a été préservée des désordres sociaux au point que seuls les districts de police de Stockholm, de Göteborg et de Malmö disposent d'unités anti-émeutes. La tenue du Conseil européen de Göteborg en juin 2001 a d'ailleurs jeté une lumière crue sur l'impréparation des forces de police suédoises à la gestion des émeutes. A cet égard, la réflexion sur les forces de police consécutivement aux événements de Göteborg a mis l'accent sur les aménagements techniques plus que sur les doctrines d'emploi des forces de sécurité. La Suède n'a donc pas modifié son modèle institutionnel de forces de sécurité d'autant plus que $75 \%$ des Suédois s'estiment satisfaits de leur force de police et que $53 \%$ considèrent que la police a bien fait son travail lors du Conseil européen de Göteborg"22. Les conclusions de "comité de Göteborg », la commission parlementaire constituée pour formuler des propositions au regard des défaillances des forces de police, sont à cet égard exemplaires. Le comité indique, d'une part, qu'une "analyse des causes fondamentales révèle que la situation concerne en premier lieu des questions qui doivent être résolues politiquement à l'intérieur de nos structures démocratiques »23. Il considère d'autre part que : « la base pour la tactique de la police doit être la non-confrontation et que les officiers de police doivent tendre à parvenir à une désescalade d'une situation potentiellement violente $»^{24}$.

De fait, l'intégration de forces de police à statut militaire dans la force civile européenne constitue pour les autorités suédoises une dénaturation de leur projet. Plus encore, la présence de forces de police à statut militaire est considérée comme une source potentielle de tensions et de violence vis-à-vis des populations locales. La Suède a entamé très tôt la diffusion de son modèle institutionnel comme en témoigne l'organisation des forces de police de Saint-Pétersbourg par des officiers de police suédois en 1893, des forces de police perses de 1910 à 1920 ainsi que des forces de police éthiopiennes de 1946 à 1949. Cette tradition se poursuit dans le cadre des programmes de contribution à la mise en œuvre de forces de police financés par l'Agence suédoise de développement international (SIDA) qui ont été conduits en Afrique du Sud ou dans les pays baltes. Par ailleurs, la conception suédoise des opérations extérieures de police se fonde sur le triptyque des missions UNCIVPOL (United Nations Civilian Police) : surveillance, éducation et conseil. Depuis la première mission UNCIVPOL, initiée à Chypre en 1964, des officiers de police suédois ont participé à 24 forces multinationales de police sous mandat de l'ONU ou de l'OSCE. Les responsables des forces de police et du ministère de la justice - ministère de tutelle des forces de police -, ainsi que les représentants des partis de gauche mettent ainsi l'accent sur la constitution de forces de police locales sous la supervision des officiers de police suédois ainsi que sur la conduite d'enquêtes criminelles sans jamais mentionner les opérations de gestion 
d'émeutes. Bien plus, ils redoutent que les missions de police de l'Union européenne n'évoluent en direction de missions d'opérations de maintien de l'ordre en zones non pacifiées qui ne correspondent pas à leur doctrine d'emploi des forces de police.

71 De même que le soldat suédois doit être un soldat de la paix dans les opérations extérieures, l'officier de police suédois doit être utilisé pour former ou reconstituer une communauté de sécurité et non pour réprimer des manifestations d'après les officiels suédois. Il est ainsi logique que les Suédois prônent l'envoi de forces civiles en amont et en aval des conflits dans la mesure où ces dernières doivent permettre la consolidation de la communauté de sécurité ou sa reconstruction ${ }^{25}$. Il apparaît que cette conception de l'emploi des forces civiles est la résultante du processus de constitution du modèle institutionnel des forces de sécurité et in extenso de la société suédoise conçue comme un modèle exportable de communauté de sécurité. Alors que la promotion de ce modèle était opérée par le ministère des Affaires étrangères suédois au nom de la neutralité au cours de la guerre froide, l'emploi de la référence politique au concept juridique de la neutralité est devenu nettement plus difficile depuis que la Suède est membre de l'Union européenne. Aussi est-ce par le biais du concept de gestion de crises que la Suède promeut ce modèle aujourd'hui ${ }^{26}$. Cela est d'autant plus aisé que le concept de gestion de crises conserve les mêmes propriétés de plasticité politique que le concept de neutralité. Les circonvolutions rhétoriques du nouveau concept stratégique suédois ne sauraient masquer la continuité de la perception des questions de sécurité en Suède. Ainsi le primat revient toujours aux opérations sous mandat de l'ONU fruit d'une conscience aiguë de la légalité internationale comme l'exprime le terme même de droit international en suédois ( $f$ olkrätt) qui renvoie à la notion du droit des gens. De même le recours à la violence n'est pas l'ultima ratio mais l'ultima optio des interventions internationales des forces de sécurité suédoises. A cet égard, il ne faut pas s'étonner du refus suédois d'élaborer une doctrine de gestion d'émeute de leurs forces de police dans le cadre de la force civile de l'Union européenne.

La Finlande

72 Le cas finlandais, s'il se différencie sur des détails, s'intègre dans un modèle qu'illustrent aussi les autres pays de la zone nordique présentés dans cet article.

73 Les forces de police qui se consacrent à la sécurité intérieure en Finlande forment un corps unique d'officiers de police dont le champ d'activité et les attributions sont définis par la loi. La police finlandaise dépend entièrement du ministère de l'Intérieur (Sisäasiainministeriö). Au sein du ministère, elle est rattachée à la Direction Centrale de la Police (Poliisin Ylijohto), que dirige un Poliisiniylijohtaja ${ }^{27}$ nommé en Conseil par le ministre. La Direction Centrale chapeaute les 5 districts de police régionaux, qui correspondent aux 5 grandes régions finlandaises (lä̈nit). Des districts locaux et des centres d'alerte permanents constituent le premier échelon de ce dispositif ${ }^{28}$.

Hormis la Police Nationale proprement dite, la Direction Centrale de la Police rassemble différents groupes à vocation spécialisée et nationale. La Police Criminelle Centrale (Keskusrikospoliisi, KRP) s'occupe des crimes «importants», aggravés ou nécessitant une assistance technique sur l'ensemble du territoire national. Elle couvre les crimes de sang nécessitant une enquête approfondie, la criminalité organisée, les trafics, les délits transfrontaliers, la délinquance informatique, le blanchiment d'argent, etc. Elle dispose de moyens techniques et de personnels spécialisés, et supervise le Laboratoire Technique du Crime (Rikostekninen laboratorio). La Police Mobile (Liikuva Poliisi) est une police de la route qui est en charge de la régulation du trafic et 
de la police routière sur les grands axes. Elle assure aussi la sécurité des aéroports, la sécurité aérienne et les missions d'escorte. La Direction Centrale gère également des services techniques (Centre Technique de la Police, Service des Chiens Policiers, unité VIRVE, etc.) ainsi que le Service des Etrangers et deux groupes spéciaux: les groupes "Karhu », une unité spéciale anti-terroriste, et les "groupes Jouha », une police antiémeute. Ces deux dernières formations sont composées de volontaires et rassemblées selon les besoins. Les Douanes (Tulli), enfin, ont des pouvoirs de police encadrés par la loi mais dépendent du ministère des Finances.

L'institution des Nimismies, enfin, est une particularité nordique que la Finlande partage avec ses voisins scandinaves. Ces fonctionnaires de police sont chargés de représenter l'état et la police dans des régions rurales ou isolées. Ils dirigent un " district de Nimismies ", et sont souvent le seul contact avec l'autorité policière connu des habitants. On a souvent comparé les Nimismies aux shérifs américains, mais leur formation en fait surtout des agents administratifs.

Les attributions de la police sont déterminées principalement par la loi sur la police (Poliisilaki) et ses différents amendements, dont la dernière version date de 1995. Cette loi s'inscrit dans des principes constitutionnels qui dictent la séparation des différents pouvoirs.

On voit donc que la police finlandaise ne dispose pas d'une institution semblable aux gendarmes français par exemple. Mais trois éléments sont à noter, qui sont particuliers à la Finlande et comptent quant on considère le lien entre cette police nationale et le milieu militaire en général.

Tout d'abord, la Finlande dispose d'une force particulière chargée d'assurer la sécurité de ses côtes et de ses frontières. Ces gardes-frontières/gardes-côtes (Rajavartiolaitos) sont une institution semi-militaire dupliquant l'organisation de l'armée finlandaise. Ils assurent la garde des frontières en temps de paix, contre les atteintes à l'ordre de droit commun, essentiellement la contrebande et l'immigration illégale. Ces unités, si elles sont sous la responsabilité du ministère de l'Intérieur en temps de paix, passent après un certain degré de crise sous celle du ministère de la Défense, et sont intégrées à l'armée. Ces gardes-côtes/gardes-frontières ont donc un statut particulier, mi-policier, mi-militaire. Ils participent aux opérations de maintien de la paix.

Ensuite, on peut noter que tous les officiers de police finlandais ont une connaissance du milieu militaire et de l'activité en milieu militaire. La Finlande a une armée de conscription, et l'immense majorité de chaque classe d'âge reçoit une formation militaire. Les officiers de police, quant à eux, doivent être officiers de réserve s'ils veulent entrer à l'Académie de police. On voit donc qu'ils ne sont pas dépaysés dans un environnement militarisé. Ce point nous a été souligné : les policiers finlandais savent s'adapter à un milieu militarisé, alors que les militaires ont plus de mal quand il s'agit de s'adapter à des missions ou à des façons d'agir relevant du maintien de l'ordre.

80 Finalement, les forces de police finlandaises ne sont pas isolées de l'armée. Celle-ci peut, par le système des « demandes d'aide officielle », être impliquée dans le maintien de l'ordre intérieur si la police s'estime débordée. Ce système s'intègre dans un ensemble de coopérations entre les « agences » de l'état finlandais. Les coopérations et leur nature dépendent aussi du degré de crise perçu par les autorités : la Finlande a un processus élaboré de montée en crise, et les attributions respectives de l'armée et de la police varient selon le degré de crise officiellement déclaré. Mais, en temps de paix, des précautions importantes entourent ce système: les opérations se font sous le 
commandement d'officiers de police, les militaires impliqués reçoivent des attributions de police mais ne doivent pas les outrepasser, etc. Ces demandes d'aide concernent surtout des matériels dont l'armée dispose et qu'elle peut prêter à la police pour des opérations ponctuelles. pour les opérations de maintien de la paix. Ce cadre a été créé pour des militaires, mais les policiers en civil suivent les mêmes modalités. La décision de participer à une opération appartient, sur proposition du Conseil d'Etat ${ }^{30}$, à la Présidence de la République. Celle-ci demande au ministère des Affaires étrangères de composer une force. Pour les policiers, la Direction Centrale de la police puise dans un pool de volontaires pour répondre à ces demandes ${ }^{31}$. Les candidats doivent être volontaires et avoir obtenu l'autorisation de leur chef de police locale. Les critères de sélection sont légèrement plus stricts que ceux imposés par l'ONU. Les candidats reçoivent deux semaines de formation spéciale à l'Académie de Police de Tampere, axée sur le contrôle des foules, la gestion de crise, le droit international, etc. Ils sont également testés en langues et en conduite. Quand un officier est sélectionné dans le pool de volontaires, il suit une formation au FINCENT, le Centre International des Forces de Défense Finlandaises, qui assure aussi la formation des militaires partant en opérations.

Dans le cadre actuel, il est intéressant de noter que la Finlande ne participe qu'à des actions ayant le soutien international le plus large possible: le parrainage par les Nations Unies est, par exemple, une condition essentielle de la participation finlandaise. L'histoire des participations finlandaises aux opérations internationales explique cela : la Finlande ne participe à ces opérations que si cela renforce sa sécurité en aidant à la paix générale; le pays ne peut s'engager dans des opérations «douteuses ». Un pays comme la Finlande a tendance à souhaiter se trouver dans le courant principal des relations internationales, sous l'égide d'une autorité internationale reconnue et respectée. Enfin, on constate que les quotas légaux de soldats envoyés en maintien de la paix ne s'appliquent pas aux policiers en civil.

La participation de la Finlande en policiers civils s'est développée avec les opérations en ex-Yougoslavie. La nature très particulière du maintien de la paix et de la reconstruction en Bosnie-Herzégovine ou au Kosovo a engendré le besoin de spécialistes de maintien de l'ordre interne. Le premier policier finlandais a été déployé 
au Mozambique en 1994, et 150 officiers ont suivi, dans différentes opérations parrainées par l'ONU (Albanie, Serbie, Macédoine, etc.).

Les résultats de ces engagements par la Finlande de policiers civils sont à plusieurs niveaux. Il est certain que la Finlande et les Finlandais ont acquis sur le terrain une expérience précieuse du maintien de la paix civile. Les qualités de leurs personnels et leur capacité à travailler en environnement militaire ont été démontrées. Les contacts entre militaires et policiers civils ont été bons, malgré quelques problèmes : si nous avons noté que les policiers finlandais sont habitués aux conditions militaires, la réciproque n'est pas toujours vraie. L'importance du contact avec les populations, qui est une part centrale du travail de police, n'est pas toujours bien comprise par les militaires.

Il nous est apparu tout aussi clairement que, après les expériences de l'ex-Yougoslavie, la volonté politique de renforcer la participation finlandaise en policiers civils existe bien en Finlande. On parle ainsi de remplacer certaines unités militaires par des policiers civils. Les autorités politiques finlandaises visent à remplir les critères de Feira définis à la fin 2001, et on table au ministère des Affaires étrangères sur le déploiement de 70 policiers en 2003. Certains acteurs considèrent aujourd'hui qu'on va lentement vers une augmentation des participations. Du côté des volontaires dans la police, il est clair aussi qu'ils ne manquent pas. La sélection est assurée au niveau de la Direction Centrale de la Police, essentiellement par le Commissaire Principal Matti Launiainen.

Des problèmes existent toutefois. Ils sont essentiellement de nature financière, avec des budgets consacrés aux participations civiles très inégaux. Les problèmes de ces participations sont différents de ceux posés par un envoi de militaires, et les fonds sont parfois mal dirigés. Mais d'autres problèmes existent. Les officiers de police, par exemple, ne voient pas souvent leur expérience du travail en environnement international validée par une avancée de carrière ou par un poste adapté à leur expérience. Les problèmes de salaire sont aussi importants: le fait de travailler à l'étranger assure un revenu plus élevé, mais le retour en Finlande signifie souvent le retour à l'ancien salaire On constate donc que le système en général reste encore prévu surtout pour des soldats. Dans la police finlandaise, on proteste aussi en sourdine contre l'envoi d'officiers de qualité à l'étranger alors que les services manquent de personnel en Finlande. On essaie toutefois, à Helsinki, de renforcer les échanges entre acteurs et les coopérations entre les différents services, Affaires étrangères, Direction Centrale de la Police, armée, etc. C'est le sens par exemple des travaux du groupe de travail interservices qui a fonctionné entre août et décembre 2001.

Concernant les opérations internationales actuelles liées aux problèmes posés par les suites du 11 septembre 2001, les Finlandais ont participé militairement à la force de maintien de la paix en Afghanistan. Toutefois, en ce qui concerne notre sujet, on peut noter qu'aucun policier en civil n'a été déployé à Kaboul par les Finlandais. De leur côté, les problèmes liés aux suites de la guerre en Irak touchent la Finlande à la fois dans le débat intérieur et extérieur. Le problème intérieur nous concerne moins ici $^{32}$ que le débat qui entoure la participation éventuelle de la Finlande à une force internationale en Irak. Deux positions s'affrontent, qui recoupent deux réactions à l'opération déclenchée par les Etats-Unis. L'échange par journaux interposés qui a opposé récemment Erkki Tuomioja, ministre des Affaires étrangères, et Paavo Lipponen, ex-Premier ministre et Président du Parlement, est symbolique de ce débat ${ }^{33}$. 
On voit qu'il existe des ferments d'opposition à une participation finlandaise dans le cadre d'une éventuelle force en Irak (Tuomioja). Mais certains estiment aussi que, une fois l'opération lancée, il en va de la crédibilité de la Finlande que celle-ci y participe (Lipponen). Une participation finlandaise à une force en Irak signifierait dans tous les cas des évolutions importantes dans la position internationale de la Finlande. Dans l'immédiat, la seule chose que l'on puisse dire est que la participation finlandaise à une telle force dépend de la bénédiction de l'ONU. Si l'on considère déjà les grandes réserves suscitées à Helsinki par l'opération armée en Irak, on voit mal la Finlande engager des forces sans le blanc-seing du Conseil de Sécurité. Pour ce qui est de notre sujet, et après l'expérience de l'Afghanistan, il est peu probable, considérant les conditions irakiennes, que des policiers civils participent à une éventuelle force.

91 On pourrait enfin évoquer brièvement les réactions des autorités finlandaises à la situation de sécurité révélée par les attentats du 11 septembre 2001 aux Etats-Unis. Les Finlandais sont conscients du risque d'attentats, même si, en dehors de quelques sites «sensibles» (l'ambassade des Etats-Unis à Helsinki par exemple), les mesures de sécurité n'ont pas été renforcées de manière significative. On considère en Finlande que le risque dans le pays est minimum, mais on manifeste une volonté de se tenir prêt à toute éventualitée ${ }^{34}$. Les autorités finlandaises ont ainsi suivi le courant européen visant à adapter les législations anti-terroristes, ainsi que les dispositifs déjà existants (une loi est en préparation pour élargir le dispositif des "demandes d'aide officielle», par exemple). Le récent conflit en Irak a encore alimenté ce débat.

Les attentats du 11 septembre 2001 ont-ils apporté des modifications aux compétences de la police en termes de sécurité intérieure?

C'est peut-être en Finlande que les changements ont été les plus importants mais ceuxci doivent être relativisés. La Finlande a suivi la tendance remarquée dans les grands pays européens d'un renforcement des moyens et de la législation anti-terroristes. Il s'agit tout d'abord d'un renforcement général des contrôles et de la surveillance des points sensibles, de l'adoption d'une législation plus sévère, et enfin de la création d'un groupe de travail chargé de coordonner l'action des différents services finlandais en cas d'attentat terroriste en Finlande. La Finlande n'a toutefois pas d'équivalent au Plan Vigipirate, mêlant militaires et policiers dans des patrouilles de surveillance. Ce plan n'a pas plus d'homologue au Danemark, en Suède et en Norvège, pays dans lesquels la législation anti-terroriste a toutefois été renforcée, tout comme la surveillance de quelques bâtiments officiels (mais rapidement levée). Encore une fois, dans aucun des quatre pays nordiques, la répartition des tâches entre la police et l'armée n'a été modifiée depuis le 11 septembre 2001.

En ce qui concerne les opérations extérieures et bien que la prise en compte d'une dimension civile de la gestion des crises au sein de l'Union européenne soit le fruit d'une initiative conjointe finno-suédoise, une certaine inquiétude se fait sentir sur l'implication des officiers de police nordiques, dont les autorités ont une conception différente de l'emploi de cette force civile de celle pratiquée par des pays disposant d'unités de police à statut militaire. Une de ces inquiétudes relève de la chaîne de commandement de la force civile : les pays nordiques estiment nécessaire de doubler l'organisation et la chaîne de commandement militaire par une organisation et une chaîne de commandement civil qui leur seraient en tous points similaires. La dépendance envers une chaîne de commandement militaire est redoutée des policiers nordiques qui s'inquiètent du manque de flexibilité de la supervision militaire. Alors 
que les policiers nordiques connaissent relativement bien le monde militaire, la réciproque n'est pas tout à fait vraie. En effet, le niveau de compréhension que les militaires peuvent avoir des exigences et de la culture policière peut paraître insuffisant.

La sécurité des pays nordiques contre toute menace extérieure est assurée par les forces armées. Les forces armées nordiques ont su développer un grand savoir-faire dans les opérations extérieures.

Si les forces armées suédoises, bien qu'elles participent à la KFOR ne semblent pas préparées à conduire des opérations de maintien de l'ordre ou de gestion des foules, les forces armées des trois autres pays nordiques ont quant à elles formé et préparé leurs hommes à intervenir face à des populations civiles hostiles. Cette préparation à la gestion des foules ou au contrôle des masses s'est faite de façon tout à fait pragmatique, aucun pays n'ayant envisagé de voir ses soldats faire du maintien de l'ordre.

Mais pour les quatre pays nordiques, tant les pratiques discursives, que les habitudes ou l'opinion de la population semblent peser lourdement sur ces questions. Il semblerait que la police soit opposée à une quelconque intervention de l'armée afin de rétablir l'ordre dans le territoire national. La police avance les arguments de sa popularité auprès des populations et de son respect du droit. L'armée ne tient pas non plus à être intégrée dans le jeu de la sécurité intérieure. Les autorités militaires affirment que l'armée n'est pas faite pour cela. Celles-ci ajoutent toutefois que si l'ordre devait leur en être donné, elles y obéiraient.

Les opinions publiques dans les pays nordiques sont puissantes. Les participations à des opérations internationales peuvent être sources de tiraillements. Les quatre pays nordiques sont certes de grands contributeurs aux opérations de maintien de la paix de l'ONU. Mais l'évolution de ces opérations en opérations de rétablissement de la paix, la participation à des opérations (dans le cadre de l'OTAN) de coalitions ad hoc comme en Irak, et non plus de l'ONU et la possible participation, à l'avenir, à des opérations dirigées par l'Union européenne, constituent des points de friction. Le risque, dans une opération extérieure difficile et menée dans le cadre de l'Union européenne, serait d'intégrer des éléments nordiques. L'éventualité que ces unités soient retirées par leurs gouvernements en cas de durcissement des opérations et de pertes humaines n'est pas à exclure, surtout si les opinions publiques s'en émeuvent. Or ces retraits pourraient à leur tour mettre en question la légitimité même des interventions extérieures par une crise qui affecterait durablement la capacité d'intervention extérieure des pays membres de l'Union européenne.

\section{NOTES}

1. Cet article prend appui sur une étude réalisée pour la Délégation aux affaires stratégiques du ministère de la Défense, intitulée : La participation des militaires aux questions de sécurité intérieure et leurs liens avec les policiers des pays qui n'ont pas 
de police à statut militaire. Le cas des pays nordiques : Norvège, Suède, Finlande et Danemark (2002).

2. . Meyzonnier P., Les forces de police dans l'Union européenne, Paris, IHESI, L'Harmattan, 1994, p. 82.

3. . The National Commissioner of the Danish Police, The Danish National Police College, Copenhague, 2001, p. 15.

4. . Jyllands-Posten, 22 mai 2002, confirmé par l'ensemble de la presse danoise le 2 juillet 2002.

5. . Entretiens avec les autorités de police danoises.

6. . Entretiens avec les autorités de police danoises.

7. . Tougher tasks.

8. . Entretiens avec les autorités de police danoises.

9. . « They prefer to be sent in more combat missions ». A ce titre, le parlement a approuvé la participation des troupes danoises à la coalition en Irak ( 150 hommes), puis à la force de stabilisation (380 hommes). Or $56 \%$ des Danois étaient alors opposés à toute participation du Danemark à la guerre contre l'Irak (sondage d'opinion de Vilstrup Research du 18 mars 2003).

10. . Ove Høegh-Guldberg, « Lessons learned from the IFOR Deployment, Danish Engagement in the Former Yugoslavia ", Nato's sixteen nations and partners for peace, Special issue 1997, pp. 82-84.

11. . Ibid. p. 83.

12. . Entretiens au ministère danois de la Défense.

13. . « Those guys from Jütland are tall, calm, they don't talk much and they don't get easily excited».

14. . Entretiens réalisés au ministère danois de la Défense.

15. . Ainsi, la Norvège souhaite retirer ses soldats affectés en Afrique au sein de missions de l'ONU pour pouvoir les redéployer en Irak.

16. . Voir loi de réquisition du 19 mai 1933, article 8, \$6, in Smith C., « Militaere maktmidler overfor norske borgere », ministère de la Défense, 1984.

17. . Smith C., ibid.

18. . Le 15 février 2003 à Oslo, 60000 personnes ont manifesté contre une guerre en Irak.

19. . Deutsch K. W., Political Community and the North Atlantic Area, Princeton, Princeton University Press, 1957, p. 5.

20. . Undersökningskommission om skotten i Ådalen, Stockholm, 1933. Les conclusions sont disponibles en suédois à l'adresse internet suivante :

http://www.skalman.nu /adalen/epilog.htm.

21. . Lindqvist H., Historien om Sverige. Drömmar och verklighet, Stockholm, Norstedts, 2000, pp. 332-340.

22. . Bennulf M., Opinion 2001. Nya Hot och risker, Stockholm, Utgiven av Styrelsen för psykologiskt försvar, 2001, p. 9.

23. . Götebörgskommittén, SOU 2002 :122 Summary, Stockholm, 2002, p. 7.

24. . Ibid., p. 9.

25. . Regerinskansliet, Preventing Violent Conflict-Swedish Policy for the 21st Century, Government Communication 2000/01 :2, Stockholm, 2000.

26. . Regerinskansliet, Svensk utrikespolitik. Utdrag ur budgetpropositionen för 2002, Stockholm, 2001, p. 17. 
27. . L'actuel directeur est Reijo Naulapää. L'actuel ministre de l'Intérieur finlandais est Kari Rajamäki.

28. . Les districts de police de Helsinki et de l'archipel des Ahvenanmaa (Åland) ont une organisation particulière. Celui de Helsinki dépend directement de la Direction Centrale, tandis que celui des Ahvenanmaa a son organisation propre définie par le statut d'autonomie de l'archipel.

29. . Un audit du cabinet Balance Consulting sur les années 1998-2000 a mis le doigt sur ces difficultés financières, soulignant l'insuffisance des moyens de la police finlandaise (voir Helsingin Sanomat du 1er mars 2002).

30. . Le Conseil des Ministres finlandais. Le Gouvernement est appelé Gouvernement, mais sa réunion en Conseil constitue le Conseil d'Etat.

31. . Les gardes-côtes/gardes-frontières participent également, en tant que personnels civils, aux opérations de maintien de la paix dans lesquelles la Finlande est engagée.

32. . L'Irak a été le sujet d'une crise importante en Finlande pendant plusieurs mois. La campagne des élections législatives de mars 2003 avait vu le parti d'Anneli Jääteenmäki, utiliser des documents secrets du ministère des Affaires étrangères pour attaquer le gouvernement sortant (celui de Lipponen) sur sa politique américaine.

Mme Jääteenmäki a dû démissionner trois mois à peine après son accession au pouvoir. 33. . Tous deux membres du parti social-démocrate, Tuomioja et Lipponen sont pourtant les symboles de deux perceptions différentes des relations internationales en général et du conflit en Irak en particulier. Ils se sont opposés, par journaux interposés, sur le thème de la participation finlandaise à une éventuelle force en Irak. Voir par exemple l'éditorial du Helsingin Sanomat du 17 juin 2003 (« Lipponen jatkaa keskustelua Suomen Irakin-politiikasta »), ainsi que l'article sur le sujet dans l'édition du 15 juin («Lipponen : Ulkoministeriössä vahingollisia USA-asenteita »).

34. . La Finlande a cru pendant quelques heures être la victime d'un attentat terroriste, le 11 octobre 2002, quand les matières détonantes, transportées par un adolescent amateur d'explosifs, ont explosées accidentellement dans un centre commercial de la banlieue de Helsinki. L'accident fit plusieurs morts et des dizaines de blessés.

L'ambiguïté de la Finlande dans les problèmes de terrorisme vient du fait que les Finlandais perçoivent le danger terroriste, se préparent, mais ne ressentent pas leur pays comme une cible particulière. Les autorités se préoccupent des possibilités pour la Finlande de devenir un lieu de refuge pour terroristes, un havre où préparer des attentats. Ce problème est suivi par les forces de police finlandaises, en particulier la SuPo, la Police de Protection chargée du contre-espionnage. Le terrorisme est en tous cas un sujet de choix pour les journaux d'information. L'édition 24/2003 de Suomen Kuvalehti revient ainsi sur le terrorisme et la Finlande, avec une couverture alarmante à souhait mais aussi un entretien intéressant avec Seppo Nevala, le directeur de la Police de Protection. 
INDEX

Index géographique : Europe du Nord

Mots-clés : coopération internationale, régionalisme et régionalisation, sécurité, sécurité intérieure 